(Aus dem physiologischen Laboratorium in Rostock.)

\title{
Die Helligkeit des Schwarz und Weiss.
}

\author{
Von
}

\section{Hermann Aubert.}

Für Versuche zur Bestimmung der Unterschiedsempfindlichkeit habe ich früher ${ }^{1}$ ) untersucht, wie viel mehr Licht von weissem Papier als von mattem schwarzen Papier reflectirt wird, oder wie viel mal heller weisses Papier ist, als mattes schwarzes Papier, und habe damals gefunden, dass das angewendete sehwarze Papier 57 mal stärker beleuchtet werden miisse, als das angewendete weisse Papier, wenn die beiden Papierflächen auf dunklem Grunde gleich hell erscheinen sollen. Diese Angabe ist von Posch ${ }^{2}$ ) dahin missverstanden worden, dass „Weiss" nur etwa 60 mal heller sei, als "Schwarz", und da ich mich sehr deutlich ansgedrïckt hatte, so habe ich jenen Ausdruek nur als einen lapsus calami ansehen können ${ }^{3}$ ). Kürzlich aber hat von Kries ${ }^{4}$ ) diesen Irrthum wiederholt und darauf woitere Schlïsse gegründet. Es scheint mir daher geboten, die Sachlage aufzuklären.

Wir machen im alltäglichen Leben die Erfahrung, dass sogenannte schwarze Objecte ein Schwarz von sehr verschiedener Tiefe zeigen, dass schwarzes Tuch, schwarze Seide grau erscheint neben schwarzem Sammet, eine Erscheinung, welche darauf beruht, dass

1) A ubert, Physiologie der Netzhaut, 1865, p. 72.

2) Posch, Ueber Sehschärfe und Beleuchtung. Archiv für Augenund Ohrenheilkunde, V, 1876, p. 21.

3) Jahresbericht für Ophthalmologic, VII, 1876, p. 132.

4) D. von Kries, Die Gesichtsempfindungen und ihre Analyse, 1882, p. 58. (Die Abhandlung von v. Kries ist auch dem Jahrgange 1882 des Archivs für (Anat. u.) Physiologie als Supplementband einverleibt worden.) 
von Tuch oder Seide mehr Licht zurïckgeworfen wird, als von schwarzem Sammet. Für gefirnisste blausehwarze Scheiben, oder Scheiben, auf denen das Blauschwarz noch nicht getrocknet war, hat 01 e $B u_{11}^{1}$ ) gefunden, dass sie 192 mal weniger Licht reflectirten, als eine weisse Scheibe, wobei er sich des von mir angegebenen Verfahrens bediente. Wenn trotzdem von Kries sagt, "dass das hellste Weiss etwa $57 \mathrm{mal}$ so hell sein soll, als das dunkelste schwarze Papier, welches man erhalten kann", und dann die Zahl $57 \mathrm{zu}$ Grunde legt zur Berechnung der unterscheidbaren Helligkeiten, in denen wir Körperfarben zu sehen pflegen, so muss er zu einer zu niedrigen Zahl gelangen, denn schwarzer Sammet ist ja offenbar viel dunkler, als mattes schwarzes Papier und wenn man viele Sammetsorten neben einander ausbreitet, so nimmt man an ihnen sehr verschiedene Grade der Schwärze wahr.

Es war mir bei meinen früheren Versuchen nicht gelungen, die Helligkeit sehwarzen Sammets im Vergleich mit weissem Papier zu bestimmen, da das Kerzenlicht sich zu schwach zeigte, und ich konnte nur so viel feststellen, dass weisses Papier wohl uiber 300 mal heller sein müsse, als schwarzer Sammet. Das hellere Licht, welches die Warmbrunn-Quilitzsche Beleuchtungslampe (Catalog von Warmbrunn und Quilitz. Abth. Physik. Nr. 936) giebt, bei welcher ein Strom von Leuchtgas mit erhitzter, comprimirter Luft gemengt gegen eine beschränkte Stelle eines Kalkcylinders geblasen wird, schien für diese Bestimmungen günstiger zu sein. Die Anordnung der Versuche war folgende: in einem gänzlich finsteren, ganz und gar schwarz angestrichenen Zimmer wird eine Scheibe weissen Cartonpapiers von etwa $20 \mathrm{~cm}$ Durchmesser an einem grossen, ebenen Sttick Sammet von $1 \mathrm{~m}$ Höhe und etwa $1 / 2 \mathrm{~m}$ Breite festgesteckt und senkrecht an die schwarze Wand gestellt. Die Beleuchtungslampe befindet sich in 2-3 m Entfernung davon. In ziemlich grosser Nähe der Lampe wird das zu untersuchende, schwarze Object möglichst eben und faltenlos ausgespannt so aufgestellt, dass von dem Statif, auf welchem es befestigt ist und welches mit schwarzem Sammet bedeckt wird, nichts zu sehen ist. Der Beobachter befindet sich ein oder mehrere Meter hinter der Lampe, welche durch einen, Schirm verdeckt

1) Ole B. Bull, Studien über Lichtsinn und Farbensinn. v. Graefe, Archiv für Ophthalmologie, Bd. XXVII, 1., 1881, p. 63. 
sein muss, und sieht, um nicht durch irgend welche Reflexe gestört zu werden, durch eine innen geschwärzte Röhre so nach der grossen schwarzen Sammetfläche, dass die kleine schwarze Scheibe in die nächste Nähe der weissen Papierscheibe projicirt wird. Dann wird die kleine schwarze Scheibe von einem Gehtilfen dem Lichte genähert oder von ihm entfernt, bis die beiden Scheiben auf der Sammetfläche genau gleich hell erscheinen, bezw. die weisse Scheibe heller erscheint, als die schwarze oder umgekehrt. Ich habe auch statt der weissen Scheibe auf schwarzem Sammet einen grossen weissen Papierbogen von 3-4 $\square$-m ausgespannt und auf diese Fläche die schwarze Sammetscheibe projicirt, welche bei gleicher Helligkeit von dem Papier nicht unterschieden werden kann und daher verschwindet - doch hat sich diese Anordnung nicht zweckmässig gezeigt, da sie unsicherere Resultate giebt, als der Vergleich zweier heller Scheiben auf tiefschwarzem Grunde.

Bei aller Vorsicht und Aufmerksamkeit sind die Bestimmungen der Helligkeit schwarzen Sammets ziemlich unsicher, weil der Sammet dem Lichte sehr nahe gebracht werden muss, und bei der intensiven Beleuchtung die Sammetfläche nie ganz gleichmässig: hell erscheint, sondern hellere und dunklere Partieen auftreten, wabrscheinlich in Folge von Schiefstellung einzelner Gruppen von Fäden; jedes Stäubchen wirkt natürlich auch äusserst störend.

Der schwarze Sammet war der schwärzeste, den ich zwisehen einer grossen Anzahl verschiedener Sammetsorten finden konnte; er hatte keine merkliche Blaufärbung. Es ist bei allen derartigen Vergleichsbestimmungen von Helligkeiten und Farben durchaus nothwendig, nicht starr auf das eine oder andere Object viele Sekunden lang hinzublieken, sondern nur ganz kurze Zeit die Objecte anzusehen und den Blick wandern zu lassen, oder die Augen abwechselnd Sekunden lang zu schliessen. Am besten ist es ferner, wenn man für die Ferne das Auge einstellt.

Trotz alledem sind die Schwankungen der Distanzen, in denen die schwarze Sammetscheibe ebenso hell erscheint, als die weisse Papierscheibe, sehr gross, denn eine scheinbare Gleichheit der beiden Scheiben fand sich bei einer Entfernung der weissen Papierscheibe von der Lichtquelle $=2720 \mathrm{~mm}$ und einer Entfernung der schwarzen Sammetscheibe $=125 \mathrm{~mm}$, woraus sich die Helligkeit des weissen Papiers 473 mal grösser berechnet, als die des schwarzen Sammets. Die Scheiben wurden aber auch als 
gleich hell angesprochen, wenn die weisse Scheibe $2290 \mathrm{~mm}$, die Sammetscheibe $90 \mathrm{~mm}$ von der Lichtquelle entfernt, die Sammetscheibe also 645 mal stärker, als die weisse Papierscheibe beleuchtet war. Erst bei einer 924 mal stärkeren Beleuchtung erschien die Sammetscheibe heller, bei einer 360 mal stärkeren Beleuchtung deutlich dunkler, als das weisse Papier. Bei Beleuchtung mit einer gewöhnlichen Gasflamme erschien die Sammetscheibe ebenso hell wie die Papierscheibe, wenn sie 730 mal stärker beleuchtet war.

Wenn diese grossen Schwankungen die Bestimmung der Helligkeit des schwarzen Sammets schon sehr unsicher erscheinen lassen, so werden wir bald noch eine andere Erfahrung mitzutheilen haben, wodurch dieselbe noch unsicherer wird. Es wurden zunächst noch andere schwarze Objecte nach dieser Methode, theils mit Kalklicht, theils mit Gaslicht untersucht und auch bei ihnen grosse Schwankungen gefunden.

So erschien eine mit'schwarzem wollenen Stoffe (Tibet) iberzogene Scheibe unter gleichen Versuchsbedingungen bei 112 mal und bei 60 mal stärkerer Beleuchtung ebenso hell, wie das weisse Papier. Die zwischen diesen Extremen gefundenen Werthe sind $100 ; 92 ; 88 ; 86$. - Derselbe Stoff sieht grau aus, wenn er bei gewöhnlicher Tagesbeleuchtung oder im Sonnenschein auf schwarzen Sammet gelegt wird.

Für eine über Petroleumflamme berusste Blechscheibe schwankten die gefundenen Werthe am wenigsten; sie erschien ebenso hell wie die weisse Scheibe, wenn sie 59 mal und wenn sie 49 mal stärker beleuchtet wurde. Es ist nothwendig, die Scheibe so zu berussen, dass sie nicht in die Flamme eintaucht, sondern nur den tiber der Flamme sich bildenden Russ annimmt, denn da, wo sie von der Flamme getroffen wird, bilden sich hellere Stellen.

Endlich wurde die Helligkeit matten schwarzen Papiers, und zwar derselben Sorte, welche ich vor zwanzig Jahren benutzt hatte, untersucht, aber fast durchgängig höhere Werthe für die Menge des von ihm reflectirten Lichtes, sowohl bei Kalklicht als bei Gaslicht gefunden: bei Kalklicht musste das schwarze Papier 31 mal, bei Gaslicht 60 bis 30 mal stärker beleuchtet werden, um ebenso hell, wie das weisse Papier zu erscheinen. $\mathrm{Ob}$ ein Ausbleichen oder eine Vermehrung der Helligkeit durch Reibung oder durch Bestäuben 
stattgefunden hat - oder ob die jetzigen Bestimmungen wegen der absolut grösseren Helligkeit des Lichtes anders ausgefallen sind, kann ich nicht sagen, um so weniger, als die Schwankungen sehr gross sind.

In Betracht dieser für alle Objecte ganz ausserordentlich grossen Schwankungen und mithin unbefriedigenden Versuchsresultate schien es mir nothwendig, eine andere Methode zur Bestimmung der Helligkeit des Schwarz anzuwenden, und als eine solche bot sich die der rotirenden Scheiben dar. Um die Differenz zwischen der Helligkeit schwarzen Sammets und schwarzen Papiers zu messen, musste dem schwarzem Sammet auf den Maxwell'schen Scheiben ein so grosser Sector von weissem Papier beigegeben werden, dass der bei der Rotation entstehende Kranz gleich hell erschien, wie die gleichfalls rotirende schwarze Papierscheibe. Wie Maxwell das aus Farben gemischte Grau einem aus Weiss und Schwarz gemischten Grau gleich machte, so musste jetat ein aus weissem Papier und schwarzem Sammet gemischtes Grau gleich der schwarzen Papierscheibe aussehen. Die Versuche lassen sich ferner so variiren, dass sowohl dem schwarzen Sammet eine gewisse Menge Weiss, als auch dem schwarzen Papier eine solche Menge Weiss beigegeben wird, dass beide, wenn sie in schnelle Rotation gesetzt werden, gleich hell erscheinen; aus der Differenz der beigegebenen Mengen Weiss berechnet sich dann die Helligkeit des sehwarzen Papiers.

Sowohl der schwarze Sammet als auch der Tibet liessen sich iiber Erwarten gut auf eine dünne Pappscheibe, welche selr dünu und gleichmässig mit flüssigem Leim bestrichen war, festkleben, und über sie Scheiben von demselben sehr rein weissen Cartonpapier, welches bei den Reflexbestimmungen benutzt worden war, verschieben. Ein äusserer Ring aus $x^{0}$ weissen Papiers und $360^{\circ}-x^{0}$ schwarzen Sammets gebildet, soll also ein genau gleiches Grau geben, wie ein innerer ans $360^{\circ}$ Tibet oder schwarzem Papier bestehender Kranz. Bezeichnen wir den Sammt mit $\Sigma$, den schwarzen Tibet mit $\mathfrak{S}$, das matte sehwarze Papier mit $S$, das weisse Papier mit $W$, so ergeben sich folgende Gleichungen:

$$
6^{\circ} \mathrm{W}+354^{\circ} \mathrm{\Sigma}=360^{\circ} \mathrm{S} \text {. }
$$

Setzen wir die Helligkeit des schwarzen Sammets $\Sigma=0$, so ergiebt sich die Helligkeit des schwarzen Tibets $\subseteq=\frac{1}{60}$; da aber 
Sammet auch Licht reflectirt, und wir auf Grund der Reflexbestimmungen seine Helligkeit ungefähr $=\frac{1}{500} W$ setzen können, so wird $\varsigma=\frac{1}{54} W$ zu setzen sein.

2)

$$
14^{\circ} W+346^{\circ} \Sigma=360^{\circ} S,
$$

worans für $\Sigma=0$ sich findet $S=\frac{1}{26,6} W$ und für $\Sigma=\frac{1}{500} W$ sich ergiebt $S=\frac{1}{24,5} W$.

$$
9^{\circ} W+351^{\circ} \subseteq=360^{\circ} S
$$

für $\subseteq=\frac{1}{60} W$ würde $S=\frac{1}{24} W$, für $\subseteq=\frac{1}{54} W$ würde $S=\frac{1}{23} W$ sein.

Diese Gleichungen zeigen eine so grosse Uebereinstimmung, wie sie bei derartigen Beobachtungen nur irgend erwartet werden kann, und mit ihnen stimmen ebenso die andern Gleichungen, in welchen beiden Arten von Schwarz eine gewisse Menge von Weiss beigemischt war, nämlich, wenn wir $\Sigma=\frac{1}{500} W$ setzen :

4)

$$
36^{\circ} W+324^{\circ} \Sigma=23^{\circ} W+337^{\circ} S,
$$

woraus $S=\frac{1}{25} W$.

5) $38^{\circ} \mathrm{W}+322^{\circ} \mathrm{\Sigma}=25^{\circ} \mathrm{W}+335^{\circ} \mathrm{S}$, woraus $S=\frac{1}{25} W$.

6) $59^{\circ} W+301^{\circ} \Sigma=47^{\circ} W+313^{\circ} \mathrm{S}$, woraus $S=\frac{1}{25} W$.

7) $\quad 126^{\circ} \mathrm{W}+234^{\circ} \mathrm{\Sigma}=117^{\circ} \mathrm{W}+243^{\circ} \mathrm{S}$, woraus $S=\frac{1}{25,6} W$.

8) $177^{\circ} \mathrm{W}+183^{\circ} \mathrm{\Sigma}=170^{\circ} \mathrm{W}+190^{\circ} \mathrm{S}$, woraus $S=\frac{1}{25,3} W$.

Als Mittelzahl aus allen diesen Gleichungen 1) bis 8) erbält $\operatorname{man}$ also $S=\frac{1}{25} W$ und $\subseteq=\frac{1}{54} W$. 
Die Werthe sind aber grösser, als die nach der Reflexmethode gefundenen höchsten Werthe. Worauf die Verschiedenheit der Resultate beruht, habe ich nicht ausfindig machen können; jedenfalls glaube ich aber den Versuchen mit den rotirenden Scheiben im vollen diffusen Tageslichte mehr Vertrauen schenken zu müssen, da bei ihnen Adaptationseinflïsse und subjective Störungen ausgeschlossen sind, ausserdem die grosse absolute, und wie ich früher nachgewiesen habe, günstigste Helligkeit des diffusen Tageslichtes eine genauere Wahrnehmung von Helligkeitsunterschieden gestattet, und endlich die für das schwarze Papier gefundenen Werthe durchweg mit einander ibereinstimmen.

Wenn, was mir nicht unwahrscheinlich ist, der Helligkeitswerth für $S$ im Tageslichte grösser ist, als für $S$ bei künstlicher Belenchtung, so ist von mir früher bei Berechnung der maximalen Unterschiedsempfindlichkeit ${ }^{1}$ ) der Werth $S=\frac{1}{57} W$ zu niedrig gewesen; wenn ich in die dortigen Gleichungen den Werth $\frac{1}{30}$ einsetze, so wïrde die Untersehiedsempfindlichkeit im Maximum nicht $\frac{1}{186}$, sondern statt dessen $\frac{1}{192}$ betragen haben, was in Beriicksichtigung: des von Dobrowolsky ${ }^{2}$ ) gefundenen Resultates, dass seine Unterschiedsempfindlichkeit für gelbes Licht sogar den Werth von $\frac{1}{272}$ erreicht, sebr möglich ist.

Wenn nun offenbar sehr verschiedene Mengen Licht von sogenannten schwarzen Objecten zurückgeworfen werden, und von schwarzem Sammet jedenfalls mindestens 300 mal weniger Licht zurückgeworfen wird, als von weissem Papier - - wenn ferner von weissem Papier sehr viel mehr Licht zurïckgeworfen wird, wenn die Sonne darauf scheint, als wenn es von diffusem Tageslichte beleuchtet ist - wenn ferner für weisses Papier, welches von der Sonne beschienen wird, noch eine Unterschiedsempfindlichkeit von

1) Aubert, Physiologie der Netzhaut, 1865, p. 76.

2) Dobrowolsky, Ueber die Empfindlichkeit des Auges gegen verschiedene Spectralfarben etc. v. Graefe, Archiv für Ophthalm., Bd. XVIII, 1., p. 68 . 
mindestens $\frac{1}{100}$ vorhanden ist ${ }^{1}$ ), so kann man weder mit vou Kries das hellste Weiss, welches von Körpern zurückgeworfen wird, nur 57 mal heller setzen als das tiefste Schwarz, welches wir an $\mathrm{Ob}$ jecten sehen, noch mit ihm die Unterschiedsempfindlichkeit innerhalb der Grenzwerthe von weissem und schwarzen Papier $=\frac{1}{50}$ annehmen. Indem von Kries diese Zahlen für die Berechnung der Anzahl unterscheidbarer Helligkeiten zu Grunde legt, findet er dieselbe $=204$, indem er den Logarithmus der Grenzzahl 57 dividirt durch den Logarithmus der wahrnehmbaren Helligkeitsunterschiede $=\left(\frac{51}{50}\right)$. Nun ist aber für das weisse und schwarze Papier der Masson'schen Scheiben bei diffusem Tageslichte die Unterschiedsempfindlichkeit immer noch über $\frac{1}{100}$; setze ich die Helligkeit des weissen Papiers nur 25 mal grösser, als die des schwarzen matten Papieres, wie wir oben Gleichung 1) bis 8) gefunden haben, so würden wir nach von Kries zu setzen haben $\left(\frac{101}{100}\right)^{x}=25$, woraus $x=323$. Zu diesem Werthe wtirde aber noch hinzuzufügen sein ein Werth, der sich in gleicher Weise berechnet aus den Helligkeiten des weissen Papiers zwischen diffusem Tageslichte und directer Sonnenbeleuchtung, und den Helligkeiten, welche geringer sind, als die des schwarzen Papiers im diffusen Tageslichte; bei sehr niedriger Schätzung würden wir dieselben auch $=25$ setzen, und innerhalb dieser Helligkeitsgrenzen würde unsere Unterschiedsempfindlichkeit eine Differenz von 50 zu 51 mit Leichtigkeit auffassen; es kämen also, wenn wir $\left(\frac{51}{50}\right)^{x}=$ 25 setzen, woraus $x=162$, diese noch hinzu, so dass damit die Zahl der unterscheidbaren Helligkeiten $323+162=485$ betragen würde, während von Kries nur 204 berechnet.

Wenn ich nun auch alle die sehr starken Reductionen gelten lasse, welche von $\mathrm{Kries}$ für die Unterscheidbarkeit von Farbentönen und Sättigungsgraden macht, so würde die Zahl der unterscheidbaren Gesichtsempfindungen immer noch auf $11500.170=$ $1855000 \mathrm{~d}$. h. beinahe auf 2 Millionen zu schätzen sein, während von Kries nur auf 5-600000 kommt. Statt der "vielen Millionen"

1) Physiologie der Netzhaut, p. 78. 
unterscheidbarer Farbenempfindungen, welche ich $\left.{ }^{1}\right)$ statuirt habe, nur zwei Millionen gelten zu lassen, würde ich mich gern bequemen, da derartige Schätzungen, eben weil sie nur Schätzungen sind, keine grosse Bedeutung haben.

Wichtiger scheint es mir aber, dass der Unfang unseres Lichtsinnes festgestellt werde, was ich durch fortgesetzte Versuche zu thun vorhabe. Zunüchst lag es mir daran, eine nur für ganz bestimmte zufallige Bedingungen gefundene Zahl des Scheines za berauben, als sei sie der Ausdruck für die Grenzwerthe unseres Lichtsinnes.

\title{
Ueber die Fettresorption im Dünndarme.
}

\author{
Von
}

Th. Kawarykin,

Professor in St. Petersburg.

Hierzu Tafel II.

Ich habe gefunden, dass die Kräfte, welehe die Fette aus dem Darmlumen fangen und dieselben weiter befördern, in den Lymphzellen der adenoiden Substanz der Darmzotten gegeben sind.

Wenn man sich einen feinen Schnitt aus dem mit Ueberosmiumsäure behandelten Darmstiicke eines vor einigen Stunden gefütterten Säugethieres (ich habe Hunde, Kaninchen und weisse Ratten untersucht) fertigt und denselben behufs der rothen Kernfärbung mit Picroearmin bearbeitet, mit Alkohol entwässert, mit Nelkenöl aufhellt und in Canadabalsam einschliesst, so findet man bei mikroskopischer Untersuchung solcher Objecte den oben anfgestellten Satz bestätigt. Ich habe nur diese Methode der Anfertigung der mikroskopischen Präparate bei dieser Untersuchung

1) Aubert, Physiologische Optik. v. Graefe u. Saemisch's Handbuch der Augenheilkunde, Bd. II, 2., 1876, p. 534.

E. Pfüger, Archiv f. Physintogie. Md, XXXI. 